\title{
Análise físico-química e sensorial de doce de leite produzido sem adição de sacarose
}

\author{
Maria Patrícia Milagres ${ }^{1}$, Geruza Dias ${ }^{1}$, Mirella Araújo Magalhães², Mateus Ottomar Silva², Afonso Mota Ramos ${ }^{3}$
}

\section{RESUMO}

O objetivo foi avaliar doces de leite produzidos sem adição de açúcar. Realizaram-se três formulações: doce com adição de açúcar (A), com edulcorante sucralose (B) e doce com ciclamato, sacarina e sorbitol (C). Nos doces B e C utilizou-se a carragena. Realizaram-se análises físico-quimicas, microbiológicas, exigidas pela legislação vigente, e análise sensorial. Nas análises de acidez não houve diferença significativa entre as amostras. O teor de gordura, a atividade de água e textura das amostras não diferiram entre os doces B e C, e esses diferiram estatisticamente do A. Nas análises de $\mathrm{pH}$ houve diferença significativa entre os doces. O teor de sólidos solúveis do doce A apresentou 63 ${ }^{\circ}$ Brix, e os doces B e C, $26^{\circ}$ Brix. Na aceitação, observou-se diferença significativa para todos os atributos avaliados. Para sabor, o doce A foi mais aceito que os doces B e C, e o doce B mais que o C. Para atributo de textura sensorial e impressão global, o doce A foi mais aceito que os doces B e C, e esses apresentaram a mesma aceitação. Na análise de textura instrumental, os doces $\mathrm{B}$ e $\mathrm{C}$ não obtiveram a mesma força de resistência à penetração que o doce $\mathrm{A}$. Todas as análises microbiológicas dos doces A, B e C apresentaram-se dentro dos padrões exigidos pela legislação vigente. Doce de leite com sucralose (B) mostrou-se uma boa alternativa para substituição do doce de leite com sacarose (A), uma vez que obteve boa aceitação para o atributo sabor.

Palavras-chave: Educorantes, light, aceitação.

\section{ABSTRACT}

\section{Physiochemical and sensorial analyses of milk fudge prepared without sugar}

This study was carried out to evaluate a milk fudge recipe prepared without addition of sugar. Three formulations were prepared: milk fudge with addition of sugar (A), with the sweetener sucralose (B), and with cyclamate, saccharin and sorbitol (C). Carrageenan was used in the formulations B and C. Physiochemical and microbiological analyses required by the Brazilian legislation and sensory analysis were performed. No significant difference was found in the acidity analyses among the samples, whereas significant difference was found for $\mathrm{pH}$ among the formulations. There was no significant difference in fat content, water activity and texture between formulations B and C, which statistically differed from formulation A. Soluble solids content for formulation A was $63^{\circ}$ Brix, whereas for formulations B and C was $26^{\circ}$ Brix. There was significant difference for acceptance for all attributes under evaluation. The sensory analysis indicated a higher acceptance for the flavor of formulation, A than formulations B and C, and B was preferred to C. Sensory tests for texture and global impression indicated that formulation A was preferred to B and C, and the last two

\footnotetext{
Recebido para publicação em dezembro de 2008 e aprovado em maio de 2010

${ }^{1}$ Engenheiras de Alimento, Mestres. Departamento de Tecnologia de Alimentos, Universidade Federal de Viçosa (UFV), Av. P. H. Rolfs, s/n, 36570-000, Viçosa, Minas Gerais (MG),Brasil.mpmilagres@yahoo.com.br, geruzadias@yahoo.com.br.

${ }^{2}$ Bachareis em Ciência e Tecnologia de Laticínios. Departamento de Tecnologia de Alimentos, UFV, Av. P. H. Rolfs, s/n,36570-000, Viçosa, MG. mimimagalhaes@yahoo.com.br, mateusottomar@yahoo.com.br

${ }^{3}$ Engenheiro de Alimentos, Ph.D. Departamento de Tecnologia de Alimentos, UFV, Av. P. H. Rolfs, s/n,36570-000, Viçosa, MG. amramos@ufv.br.
} 
had the same acceptance. The fat content of formulation A was lower than the other two. In the instrumental texture analysis, formulations $\mathrm{B}$ and $\mathrm{C}$ had lower resistance to penetration than formulation A. All microbiological analyses of formulations A, B and C complied with the standards required by the legislation. Milk fudge formulated with sucralose (B) is thus a good market alternative to formulations with sucrose (A), since it had similar acceptance to common milk fudges.

Keywords: Acceptance, edulcorant, light food.

\section{INTRODUÇÃO}

O doce de leite é um importante alimento produzido e comercializado principalmente na Argentina e no Brasil. Trata-se de um produto obtido pelo cozimento de leite adicionado de sacarose, que adquire coloração, textura e sabor característicos em função de reações de escurecimento não-enzimático, sendo muito apreciado pelos consumidores. É amplamente empregado como ingrediente para a elaboração de alimentos como confeites, bolos, biscoitos, sorvetes e também consumido diretamente na alimentação como sobremesa ou acompanhado de pão, torradas ou de queijo (Demiate et al., 2001).

A indústria de alimentos, atenta ao mercado consumidor, tem desenvolvido produtos e tecnologias para a produção de alimentos com baixas calorias, mediante substituição de açúcar por edulcorantes e/ou redução no seu teor de gordura (Cândido \& Campos, 1996).

Os edulcorantes apresentam ação adoçante, podendo substituir a sacarose. Alguns desses compostos são conhecidos como edulcorantes intensos, pois fornecem doçura acentuada, sendo utilizados em quantidades muito pequenas. Geralmente são não-nutritivos ou pouco calóricos e não apresentam ação cariogênica. Entre os edulcorantes não-nutritivos, ou seja, aqueles que não são metabolizados pelo organismo, não fornecendo energia, citam-se, de acordo com Benassi et al. (2001): a sacarina, cujo poder edulcorante é cerca de 300 vezes a da sacarose, revela frequentemente gosto amargo; o ciclamato, de 30 a 50 vezes mais doce que a sacarose, apresenta sabor residual agridoce desagradável; o acesulfame-K, cerca de 180 a 200 vezes mais doce do que a sacarose, tem perfil de doçura semelhante ao da glicose; e a sucralose, obtida a partir do açúcar comum, cujo poder edulcorante pode variar de 400 a 800 vezes em relação à sacarose.

Os edulcorantes nutritivos fornecem quantidades variadas de energia, porém contribuem para a redução do valor energético dos alimentos, como é o caso do aspartame. Embora seu valor calórico seja igual ao da sacarose (4 kcal/g), é utilizado em quantidades mínimas, devido à sua intensa doçura (cerca de 180 vezes a da sacarose), o que proporciona diminuição no teor calórico. Pode ser consumido por diabéticos, mas não por portadores de fenilcetonúria, doença genética que altera o metabolismo da fenilalanina (Benassi et al., 2001).

Nenhum edulcorante é adequado para todas as aplicações, contudo suas limitações podem ser minimizadas mediante combinações entre eles. A combinação tem por objetivo aumentar o poder educorante, aproveitando o efeito de sinergismo que permite redução no nível de utilização dos edulcorantes e no custo do produto final. Além disso, a combinação visa mascarar sabores residuais e obter perfil de doçura mais próximo ao da sacarose. Um exemplo clássico é o sinergismo entre sacarina e ciclamato, que são usados frequentemente em combinação para que a sacarina aumente o poder educorante do ciclamato e esse diminua o residual amargo da sacarina (Cândido \& Campos, 1996).

De acordo com Verdi \& Hood (1993), misturas de sucralose com acesulfame-K demonstram sinergismo entre 7,2 e $9,8 \%$. Isso significa que quando os edulcorantes são misturados, guardando a equivalência do poder edulcorante de cada um, verifica-se elevação na doçura da ordem de 7,2 a $9,8 \%$.

O sucesso da sacarina pode ser atribuído a alguns fatores: preço cerca de 20 vezes menor do que o da sacarose; não é metabolizada pelo organismo, portanto não fornece calorias; não afeta os dentes; é estável e tem ampla faixa de aplicação (Higginbotham, 1983).

No Brasil existem diversos adoçantes de mesa com essa composição, e os mais vendidos possuem a proporção de duas partes de ciclamato para uma de sacarina (Wells, 1989).

As gomas são compostos poliméricos que, quando dissolvidos ou dispersos em água, formam soluções ou dispersões viscosas. Pertencem ao grupo dos hidrocoloides ou seus derivados, os quais possuem ampla aplicação como agentes espessantes e estabilizantes. Podem ser obtidos a partir de extratos de algas marinhas 
(alginatos, ágar, carragenas), extratos de sementes (locusta, guar), extratos vegetais (arábica), microrganismos (xantana, gelana) e a partir de celulose e pectina (Sanderson, 1981).

A carragena é extraída de algas vermelhas e está disponível em três tipos, chamados kappa ( $\kappa)$, iota ( 1 e e lambda $(\lambda)$. As carragenas kappa e iota são capazes de formar géis termorreversíveis (Barreto \& Beirao, 1999). Essa goma atua como emulsificante, geleificante, estabilizante e possui a capacidade de manter partículas em suspensão. O uso de carragenas é indicado para formulação de medicamentos e em diversos produtos de baixo teor calórico, como em queijos, produtos cárneos, pudins, chocolates e geleias (Cândido \& Campos, 1996).

Os mecanismos de gelificação e espessamento dos três tipos de carragena são bem diferentes. Por exemplo, kappa carragena forma um gel rígido na presença de potássio; e iota e lambda são fracamente afetadas. Na maioria dos casos, lambda é usada com a kappa em sistemas com leite objetivando obter um gel cremoso (Imenson, 1997).

A influência da temperatura é também importante fator na decisão de qual tipo de carragena deve ser usado em determinado sistema alimentício. Todas carragenas são solúveis em altas temperaturas, desenvolvendo baixa viscosidade no fluido. Entretanto, kappas e iotas conferem várias texturas aos géis de 40 a $70{ }^{\circ} \mathrm{C}$, dependendo do cátion presente. Esses géis são, obviamente, estáveis à temperatura ambiente, mas podem ser remodelados pelo aquecimento cerca de 5 a 10 graus acima da temperatura de geleificação. O resfriamento do sistema irá regelificá-lo (Imenson, 1997).

A análise sensorial é um campo muito importante na indústria de alimentos, pois contribui direta ou indiretamente para inúmeras atividades, como desenvolvimento de novos produtos, controle de qualidade, reformulação e redução de custos de produtos, relações entre condições de processo, ingredientes, e aspectos analíticos e sensoriais. No teste sensorial é muito importante a padronização das amostras. Muitas vezes o atributo que se pretende avaliar é influenciado por outros fatores, como a quantidade de amostra e a cor do produto (Pal et al., 1985).

A determinação da aceitação pelo consumidor é parte crucial no processo de desenvolvimento ou melhoramento de produtos. Os testes afetivos requerem equipe com grande número de participantes e que representem a população de consumidores atuais e/ou potenciais do produto. Entre os métodos mais empregados na medida de aceitação de produtos está a escala hedônica, em que o consumidor expressa sua aceitação pelo produto seguindo uma escala previamente estabelecida, que varia gradativamente com base nos termos gosta e desgosta (Chaves \& Sprosser, 2001).
O objetivo foi desenvolver formulações de doce de leite sem açúcar, visando oferecer ao consumidor um substituto saboroso e saudável e testar duas formulações usando diferentes combinações de educorantes, comparandoas com a formulação tradicional que emprega o açúcar quanto aos atributos sensoriais, físico-químicos, microbiológicos e rendimentos.

\section{MATERIAL E MÉTODOS}

As três formulações de doces, com adição de açúcar (doce A), com edulcorante sucralose (doce B) e com uma combinação de ciclamato, sacarina e sorbitol (doce C), foram produzidas na Fábrica de Processamento de Alimentos do Departamento de Tecnologia de Alimentos da Universidade Federal de Viçosa (UFV), Viçosa, Minas Gerais.

\section{Análises da matéria-prima}

Para a determinação da qualidade do leite usado na produção dos doces foram realizadas análises físico-químicas, segundo Brasil (2006).

As análises físico-químicas foram determinações de teor de gordura, acidez titulável, pH e crioscopia. O teor de gordura foi encontrado utilizando o método de Gerber. Para a determinação de acidez titulável foram titulados 10 gramas de leite, solução de $\mathrm{NaOH} 0,1 \mathrm{~mol} / \mathrm{L}$, utilizando-se como indicador a fenolftaleína $1 \%(\mathrm{~m} / \mathrm{v})$ neutralizada. Para o cálculo de $\mathrm{pH}$ foi empregado o método eletroanalítico. A análise de crioscopia foi realizada usando crioscopio MK 550 Tex.

\section{Elaboração dos doces}

O leite utilizado para a produção dos doces apresentou características de cor, odor e sabor normais. E sua acidez foi ajustada para $0,13 \%$ de compostos ácidos expressos como ácido lático utilizando bicarbonato de sódio comercial.

Para a formulação do doce de leite com açúcar (doce A), o leite foi adicionado em um tacho de camisa dupla aberto de aço inoxidável e dotado de um agitador mecânico. Em seguida, adicionou-se a sacarose. A mistura foi concentrada até atingir um teor de sólidos solúveis de 63 ${ }^{\circ}$ Brix, quando adicionaram-se o sorbato de potássio e o citrato de sódio. $\mathrm{O}$ produto foi resfriado até $62{ }^{\circ} \mathrm{C}$, embalado em potes de vidro e armazenado em câmara de resfriamento.

Os doces B e C foram elaborados a partir da concentração do leite com acidez ajustada e adicionado do espessante carragena em um tacho de camisa dupla aberto de aço inoxidável e dotado de um agitador mecânico.

$\mathrm{O}$ doce $\mathrm{B}$ foi produzido com o edulcorante sucralose e o C com a adição da combinação de ciclamato, sacarina e sorbitol. Os doces B e C foram concentrados até

Rev. Ceres, Viçosa, v. 57, n.4, p. 439-445, jul/ago, 2010 
atingir $26{ }^{\circ}$ Brix, de acordo com Granda et al. (2005), quando foram adicionados o sorbato de potássio e o citrato de sódio.

Para efeito deste trabalho foram usados os conceitos de adoçantes e edulcorantes propostos por Angelucci (1990). Para o ajuste da doçura dos doces de leite, os poderes edulcorantes adotados para os substitutos do açúcar foram os apresentados por Cândido \& Campos (1996), e a quantidade adicionada foi calculada de tal forma que esse poder edulcorante fosse equivalente a um doce formulado com $18 \%(\mathrm{~m} / \mathrm{m})$ de teor de açúcar.

A carragena é utilizada para ajudar a obter uma textura semelhante à do doce com açúcar. O sorbato de potássio é muito importante nesse doce, uma vez que, devido a seu reduzido teor de sólidos, pode haver problemas de contaminação microbiana.

\section{Análise sensorial}

As amostras foram avaliadas por 40 provadores nãotreinados, sendo 13 do sexo masculino e 27 do sexo feminino, com faixa etária entre 19 e 38 anos.

O teste de aceitação foi realizado no Laboratório de Análise Sensorial do Departamento de Tecnologia de Alimentos da UFV.

Foi aplicada aos provadores uma escala hedônica de nove pontos, cujos extremos correspondem a desgostei extremamente (1) e gostei extremamente (9). As amostras foram servidas de forma monádica aos provadores em cabines individuais, sob luz branca, refrigeradas $\left(5^{\circ} \mathrm{C}\right)$, em copos de $50 \mathrm{~mL}$, codificados com três dígitos, obtidos de uma tabela de números aleatórios (Pedrero \& Pangborn, 1989). Em seguida, foi solicitado aos provadores que analisassem as amostras quanto aos atributos sabor, textura e impressão global, de acordo a escala proposta.

\section{Análises físico-químicas}

Foram realizadas análises físico-químicas: acidez titulável, pH e teor de gordura seguindo a metodologia preconizada por Brasil (2006).

A determinação de sólidos solúveis foi realizada com emprego de refratômetro à temperatura de $25^{\circ} \mathrm{C}$, e o resultado foi expresso em ${ }^{\circ}$ Brix.

A textura dos doces produzidos foi determinada utilizando o texturômetro TA-HDi Texture Analyser of Stable Micro Systems, o qual mede a dureza, de forma direta, pela força máxima de penetração, em Newtons, através da célula sensor SMSP /35.

\section{Análises microbiológicas}

Foram realizadas com um dia de fabricação dos doces de leite análises de fungos filamentosos e leveduras, coliformes e presença de Staphylococcus coagulase positiva, segundo metodologia preconizada por Brasil (2003).

\section{Análise de rendimento}

O rendimento do processo das diferentes formulações de doce de leite foi avaliado empregando-se a seguinte fórmula:

$\mathrm{R}=(\mathrm{SM} \times 100) / \mathrm{SD}$

em que:

$\mathrm{R}=$ rendimento $(\%)$

$\mathrm{SM}=$ massa empregada de leite + todos os ingredientes (g); e

$\mathrm{SD}=$ massa empregada de doce $(\mathrm{g})$.

\section{Análises estatísticas}

O experimento foi conduzido com três formulações: doce com adição de açúcar (A), com edulcorante sucralose (B) e doce com ciclamato, sacarina e sorbitol (C), dispostas no delineamento inteiramente casualizado, com três repetições.

Os resultados foram submetidos à análise de variância, utilizando-se o programa SAS (Statystical Analysis System), versão 9.1, licenciado para a Universidade Federal de Viçosa. As médias dos tratamentos foram comparadas pelo teste de Tukey a 5\% de probabilidade.

\section{RESULTADOS E DISCUSSÃO}

\section{Análises da matéria-prima}

O leite utilizado para fabricação dos doces apresentou resultados para análise físico-química dentro dos parâmetros previstos pela Instrução Normativa $N^{\circ} 51$, de 18 de setembro de 2002, do Ministério da Agricultura (Brasil, 2002). Os resultados da análise físico-química da matéria prima encontram-se na Tabela 1.

\section{Análises físico-químicas dos doces}

As médias dos resultados obtidos nas análises dos doces produzidos (Tabela 2) encontram-se dentro dos padrões exigidos pela Portaria $\mathrm{N}^{\circ} 354$, de 4 de setembro de 1997 (Brasil, 1997).

A acidez titulável não diferiu significativamente entre os doces. O teor de gordura, a atividade de água e a textura instrumental não diferiram entre os doces $\mathrm{B}$ e $\mathrm{C}$, diferindo estatisticamente do doce $\mathrm{A}$. Nas análises de $\mathrm{pH}$ houve

Tabela 1. Resultados da análise físico-química da matéria prima (leite) utilizado na produção de doces de leite

\begin{tabular}{lc}
\hline Parâmetros & Valores encontrados* \\
\hline Teor de gordura & $3,0 \%+0,3$ \\
Índice crioscópico & $-0,533{ }^{\circ} \mathrm{H}+0,03$ \\
Acidez titulável ${ }^{* *}$ & $0,16 \%+0,01$ \\
\hline$\left(^{*}\right)$ Media + desvio padrão. & \\
$\left(^{* *}\right)$ Compostos ácidos expressos como ácido lático.
\end{tabular}


Tabela 2. Resultados das análises das três formulações de doces de leite: $\mathrm{A}$ = com adição de açúcar; B = com edulcorante sucralose; $\mathrm{C}=$ com uma combinação de ciclamato, sacarina e sorbitol

\begin{tabular}{|c|c|c|c|c|c|}
\hline Doces & $\begin{array}{c}\text { Acidez titulável* } \\
(\%)\end{array}$ & pH & $\begin{array}{c}\text { Textura } \\
\text { (N) }\end{array}$ & $\begin{array}{l}\text { \% Gordura } \\
(\mathbf{m} / \mathbf{m})\end{array}$ & $\begin{array}{c}\text { Sólidos solúveis } \\
\left({ }^{\circ} \mathrm{Brix}\right)\end{array}$ \\
\hline A & $0,25 a$ & $6,22 c$ & $0,521 a$ & $6,5 b$ & 63a \\
\hline B & $0,24 \mathrm{a}$ & $6,46 a$ & $0,274 b$ & $9,5 a$ & $26 b$ \\
\hline C & $0,26 a$ & $6,39 b$ & $0,267 b$ & $9,5 a$ & $26 b$ \\
\hline
\end{tabular}

Médias seguidas por pelo menos uma mesma letra (nas colunas) não diferem significativamente pelo teste de Tukey a 5\% de probabilidade. (*) Compostos ácidos expressos como ácido lático.

diferença significativa entre os doces A, B e C. O teor de sólidos solúveis no doce $\mathrm{A}$ apresentou $63^{\circ} \mathrm{Brix}$, e nos doces B e C, $26^{\circ}$ Brix (Tabela 2).

O açúcar é responsável pela menor atividade de água do doce A em relação aos demais. Mesmo adicionado de carragena, os doces B e C não conseguiram obter a mesma força de resistência à penetração que o doce $\mathrm{A}$ na análise de textura instrumental (Tabela 2).

Granda et al. (2005) desenvolveram três formulações de doces, sendo uma com sacarose, e as outras usando uma mistura de educorantes e pectina como espessante. Os resultados obtidos foram semelhantes aos do presente trabalho, com exceção da textura instrumental mais macia, em virtude do uso de carragena como espessante.

\section{Análise sensorial}

Os resultados do teste de aceitação das três amostras de doce de leite estão descritos na Tabela 3. O doce A apresentou a maior média de aceitação para todos os atributos, diferindo significativamente das demais amostras ( $p<0,05)$. Comparando os doces sem adição de sacarose, observa-se que para o atributo sabor, o doce B foi mais aceito do que o C ( $<<0,05)$. Quanto aos atributos impressão global e textura sensorial, os doces B e C não apresentaram diferença significativa $(p>0,05)$.

Os resultados da análise sensorial dos doces de leite foram semelhantes aos encontrados por Granda et al. (2005), que verificaram boa aceitação para o atributo impressão global dos doces sem açúcar. Os autores testaram três formulações, sendo a primeira com acréscimo de sacarose (doce comum: F1) e as outras com os seguintes edulcorantes: acesulfame-K e sucralose na segunda formulação (F2); e ciclamato de sódio, esteviosídeo, sacarina sódica e sorbitol na ter- ceira (F3). O agente espessante utilizado foi a pectina de baixa metoxilação nas formulações 2 e 3 . De acordo com os resultados obtidos por esses autores nas análises sensoriais, o doce formulado com sacarose obteve média 8,3, ficando entre os termos gostei muito e gostei extremamente, enquanto os doces com edulcorantes localizaram-se entre gostei ligeiramente e gostei muito, com médias 6,4 e 6,6 para as formulações 2 e 3, respectivamente.

A aceitação mais baixa em relação aos atributos impressão global e textura para os doces sem açúcar pode ser explicada pela cor mais clara e menor dureza desses. A ausência de sacarose diminui a reação de Maillard, que é uma interação química entre um aminoácido ou proteína e um carboidrato reduzido, responsável por sabor, aroma e cor dos doces de leite. A diminuição da reação de caramelização também pode justificar a menor aceitação dos doces de leite sem açúcar para o atributo textura. Nessa reação ocorre desidratação, condensação e polimerização do carboidrato; a ausência de açúcar pode diminuir essa reação.

O doce de leite produzido com sucralose foi o mais aceito para o atributo sabor dentre os doces sem sacarose. Pinto et al. (2003) encontraram resultados semelhantes. Os autores demonstraram em seu trabalho, por meio de analise sensorial de um mousse de chocolate light a existência de boa aceitação de sobremesas light adoçada com sucralose. Em uma pesquisa de mercado, Pinto et al. (2003) observaram a existência de um mercado para produtos light. Outros trabalhos que analisaram a aceitação de produtos light adoçados com diferentes educorantes também verificaram melhor aceitação do educorante sucralose (Campos, 2002; Mendonça et al., 2005)

Tabela 3. Resultados médios $(n=40)$ dos escores na avaliação da aceitação sensorial das amostras de doce de leite de diferentes formulações. A = com adição de açúcar; B = com edulcorante sucralose; C = com uma combinação de ciclamato, sacarina e sorbitol

\begin{tabular}{lcccc}
\hline Atributos & Doce A & Doce B & Doce C & DMS \\
\hline Sabor & $7,53 \mathrm{a}$ & $6,58 \mathrm{~b}$ & $5,63 \mathrm{c}$ & 0,72 \\
Textura & $7,50 \mathrm{a}$ & $4,35 \mathrm{~b}$ & $4,50 \mathrm{~b}$ & 0,59 \\
Impressão global & $7,48 \mathrm{a}$ & $5,30 \mathrm{~b}$ & $4,98 \mathrm{~b}$ & 0,59 \\
\hline
\end{tabular}

Numa mesma linha, médias com letras em comum não diferem significativamente entre si (p > 0,005); e DMS: diferença mínina significativa do teste de Tukey a 5\% de probabilidade. 
Tabela 4. Resultados médios das análises microbiológicas das amostras de doce de leite de diferentes formulações: $\mathrm{A}=$ com adição de açúcar; B = com edulcorante sucralose; C = com uma combinação de ciclamato, sacarina e sorbitol

\begin{tabular}{lccc}
\hline Microrganismos & \multicolumn{1}{c}{ A $^{*}$} & B* & C* \\
\hline Contagem-padrão em placas & $<25 \mathrm{UFC} / \mathrm{g}+0,5$ & $75 \mathrm{UFC} / \mathrm{g}+3,0$ & $103 \mathrm{UFC} / \mathrm{g}+4,0$ \\
Sthaphilococcus coagulase & $<10 \mathrm{UFC} / \mathrm{g}+0,5$ & $<10 \mathrm{UFC} / \mathrm{g}+0,8$ & $<10 \mathrm{UFC} / \mathrm{g}+0,7$ \\
Fungos e leveduras & $<25 \mathrm{UFC} / \mathrm{g}+0,8$ & $<25 \mathrm{UFC} / \mathrm{g}+1,0$ & $<25 \mathrm{UFC} / \mathrm{g}+1,2$ \\
\hline
\end{tabular}

* Medias + desvio padrão.

\section{Analises microbiológicas}

As análises microbiológicas (Tabela 4) dos doces A, B e $\mathrm{C}$ apresentaram contagem de fungos filamentosos e leveduras dentro dos padrões exigidos pela Portaria $N^{\circ} 354$, de 4 de setembro de 1997. Observou-se maior contagem total nos doces B e C, devido à maior atividade de água. Não houve crescimento significativo de fungos filamentosos e leveduras devido à adição do conservante sorbato.

Todas as amostras analisadas tiveram contagem de Staphylococcus coagulase positiva, < $10 \mathrm{UFC/g}$. As contagens de coliformes foram inferiores a $3 \mathrm{NMP} / \mathrm{g}$.

\section{Avaliação do rendimento}

Os rendimentos dos diferentes doces foram: A, 35,5; B, 29; e C, 23,6\%. O doce A apresentou maior rendimento que os doces $\mathrm{B}$ e $\mathrm{C}$.

Granda et al. (2005) relataram valores de rendimento do doce de leite formulado com edulcorante de 30 a 31\% enquanto o rendimento de doce de leite comum foi $36 \%$. A pequena diferença encontrada no trabalho de Granda et al. (2005) pode estar relacionada ao uso da pectina como espessante, que proporcionou maior rendimento dos doces de leite com edulcorante quando comparado com o do presente trabalho, que utilizou carragena como espessante.

A presença de sacarose aumentou o teor de sólidos do doce de leite comum quando comparado aos doces de leite com adição de adulcorante, aumentando assim o seu rendimento.

\section{CONCLUSÕES}

Os doces A, B e C apresentam características físicoquímicas e microbiológicas dentro dos padrões vigentes preconizados pela legislação brasileira. Portanto, podem ser consumidos seguramente.

O doce $\mathrm{B}$ tem boa aceitação para o atributo sabor nos testes dos atributos sensoriais, porém inferior ao doce $\mathrm{A}$. Não há diferença entre os doces B e C para os atributos textura sensorial e impressão global. O doce B é o mais indicado para substituição do doce de leite com sacarose.

O doce de leite $B$ apresenta-se como boa alternativa para consumidores que, por motivos de saúde, devem diminuir o consumo de açúcar, assim como para aqueles que desejam alimentos menos calóricos, por motivos estéticos.

\section{REFERÊNCIAS}

Angelucci E (1990) Legislação sobre edulcorantes no Brasil. Edulcorantes e adoçantes em alimentos: ciclo de debates. Campinas, Ital. p.1-9.

Barreto PLM \& Beirao LH (1999) Influência do amido e carragena nas propriedades texturais de surimi de tilápia (Oreochomis sp.). Ciências, Tecnologia de Alimentos, 19:183-188.

Benassi VT, Watanabe E \& Lobo AR (2001) Produtos de panificação com conteúdo calórico reduzido. Boletim do Centro de Pesquisa de Processamento de Alimentos, 19:225-242.

Brasil (1997) Portaria No 354, de 04 de setembro de 1997. Regulamento técnico para fixação de identidade e qualidade de doce de leite. Publicado no Diário Oficial da União de 08/09/1997, Seção 1, Página 19685.

Brasil (2002) Instrução Normativa $N^{\circ}$ 51, de 18 de setembro de 2002. Regulamentos técnicos de produção, identidade e qualidade do leite tipo A, do leite tipo B, do leite tipo C, do leite pasteurizado e do leite cru refrigerado e o regulamento técnico da coleta de leite cru refrigerado e seu transporte a granel.

Brasil (2003) Instrução Normativa Nº 62, 26 de Agosto de 2003. Oficializa os métodos analíticos oficiais para análises microbiológicas para controle de produtos de origem animal e água. Diário Oficial da União de 18/09 2003, Seção 1, Página 14. Ministério da Agricultura.

Brasil (2006) Instrução Normativa $N^{\circ}$ 68, de 12 de dezembro de 2006. Oficializa os métodos analíticos oficiais físico-químicos, para controle de leite e produtos lácteos, em conformidade com o anexo desta Instrução Normativa, determinando que sejam utilizados nos Laboratórios Nacionais Agropecuários. Diário Oficial da União de 14/12/2006, Seção 1, Página 8.

Campos MB (2002) Aplicação de sucralose em iogurtes e preparados de frutas para iogurtes. Food Ingredients, 19:68-71.

Cândido LMB \& Campos AM (1996) Alimentos para fins especiais: Dietéticos. São Paulo, Varela. 432p.

Chaves JBP \& Sprosser RL (2001) Práticas de laboratório de análise sensorial de alimentos e bebidas. Viçosa, UFV. 81p.

Demiate IM, Konkel FE \& Pedroso RA (2001) Avaliação da qualidade de amostras comerciais de doce de leite pastoso - composição química. Ciências, Tecnologia de Alimentos, 21:108-114.

Granda T, Ramos AM \& Teixeiras LJQ (2005) Formulação e avaliação de doce de leite em pasta sem adição de açúcar. In: XXII Congresso Nacional de Laticínios, Juiz de Fora. Anais. p.285-288.

Higginbotham JD (1983) Recent developments in non-nutritive sweeteners. In: Gremby TH, Parker KJ \& Lindley MG. Developments in sweeteners-2. London, Applied Science Publ. p.119-55.

Imenson A (1997) Thicketing and gelling agents for food. $2^{\circ}$ Ed. New York, Blackie Academic \& Professional. 342p.

Mendonça CRB, Zambiazi RC, Gularte MA \& Granada GG (2005) Características sensoriais de compotas de pêssego light elaboradas com sucralose e acesulfame-K. Ciências, Tecnologia de Alimentos, 25:401-407. 
Pal D, Sachdeva S, Singh S (1985) Methods for determination of sensory quality of foods: A critical appraisal. Journal Food Science, 32:357- 367.

Pedrero FDL \& Pangborn RM (1989) Evaluación sensorial de los alimentos - Métodos analíticos. Alhambra Mexicana, 1:103107.

Pinto EP, Teixeira AM, Sopeña LL, Rosa VP \& Luvielmo MM (2003) Sucralose no desenvolvimento de sobremesas lácteas. Boletim do Centro de Pesquisa em Processamento de Alimentos, 21:49-60.
Sanderson GR (1981) Polysaccharides in foods. Food Technology, 35:50-83.

Verdi RJ \& Hood LL (1993) Advantages of alternative sweetener blends. Food Technology, 47:94-100.

Wells AG (1989) The use of intense sweeteners in soft drinks. In: Gremby, TH. Progress in sweeteners. New York, Elsevier Applied Science, p.121-142. 